\title{
Modern External Beam Radiotherapy Techniques - Intensity Modulated Radiotherapy
}

\author{
A. ŚlLadowska \\ Maria Skłodowska-Curie Memorial Institute, Centre of Oncology, Cracow Branch \\ Garncarska 11, 31-115 Kraków, Poland
}

\begin{abstract}
External beam radiotherapy is a complex cancer radiotherapy technique involving three-dimensional conformal and intensity-modulated radiation therapy high-energy X-ray beam delivery to tumour volumes where dose distributions and treatment plan optimisation, including inverse planning, are applied. Examples of clinical treatment plans using 3D conformal and intensity-modulated radiotherapy techniques for patients with tumours located in the regions of head and neck (right tonsil carcinoma) and pelvis (prostate) were evaluated and compared. An intensity-modulated radiotherapy plan for a patient with arm cancer, untreatable with the 3D conformal radiation therapy technique, is also presented. Comparison of dose distributions showed that with the intensity-modulated radiotherapy technique dose homogeneity within the target volume was improved and dose to normal tissues and organ-at-risk structures reduced. Significant reductions in the mean and maximum doses to the spinal cord (head and neck) and rectum (prostate) were also achieved. Due to the geometrical issues, in all patients, improvement in dose distribution was evident with the intensity-modulated radiotherapy technique, especially for concave dose distributions.
\end{abstract}

PACS numbers: 87.56.-v, 87.56.J-, 87.56.N-, 87.56.jk

\section{Introduction}

Radiotherapy (i.e. radiation therapy) uses ionising radiation to eradicate cancer cells and to decrease the tumour volume. About $50 \%$ of all cancer patients are treated with radiation therapy, either alone or in combination with other types of treatment, most often surgery or chemotherapy.

In modern external beam radiation therapy, a beam of high energy (4-20 MV) X-rays or electrons $(4-25 \mathrm{MeV})$ is directed through the patient's body to the target volume, usually at several angles, in order to eradicate cancer cells in the tumour and its immediate neighbourhood. To minimise side effects, a treatment dose of about $60 \mathrm{~Gy}$ to the tumour volume is usually delivered in some 30 daily fractions of about 2 Gy each.

Before any patient receives a radiation therapy cancer treatment, careful treatment planning is undertaken. This includes detailed imaging, radiation dose calculations, and graphical review of the proposed treatment geometry. To achieve tumour control, a dose distribution is planned which has a good chance of sterilising all cancer cells without causing unacceptable normal tissue complications.

Advances in the field of medical imaging have considerably improved the process of radiotherapy treatment planning, an important element of the complex radiotherapy procedure. As a result of these changes, teletherapy techniques have evolved from conventional techniques where bone structures were used for beam setup and dose distributions were calculated manually, to new specialised computer-based planning systems involving three-dimensional (3D) reconstructions of computer tomography $(\mathrm{CT})$ images and 3D dose distribution algorithms. In two-dimensional (2D) radiotherapy single beams from one or two directions were used. Beam setups were usually quite simple and treatment plans frequently consisted of opposed lateral fields $[1,2]$. With the advent of CT imaging, which took into account axial anatomy and complex tissue contours, three-dimensional conformal radiotherapy (3D-CRT) had arrived [3]. New equipment, including digitally-controlled multileaf collimators (MLC) mounted in the gantry of the linear accelerator and computer-controlled operation of medical accelerator systems, enabled the dose distribution to be closely conformed to the tumour volume and to be varied dynamically. Intensity-modulated radiation therapy (IMRT) is a highly advanced form of 3D-CRT whereby high doses of radiation can be delivered much more precisely than in conventional radiotherapy. This is achieved not only by shaping the field geometry to conform to the given projection of the tumour volume from each direction, but also by varying the photon beam intensity within these individually shaped fields. In the IMRT technique, the field of a given radiation beam is decomposed into several small "beamlets" (subfields) and the intensity of each beamlet adjusted individually by an inverse-planning algorithm which optimises the intensity maps of fields from each direction. Intensity (fluence) maps are realised by 
dynamic movement of the MLC leaves [3, 4]. By setting the number of fields and modulating the intensity of radiation within each field, it is possible to obtain better dose conformation, including concave 3D dose distributions, unachievable by 3D-CRT technique [5].

Several radiotherapy techniques, including IMRT, are currently applied in our Centre of Oncology.

The aim of this work is to present examples of 3D-CRT and IMRT plans in order to illustrate these teletherapy techniques and their optimisation with respect to target coverage, conformity of prescribed dose volumes and critical tissue sparing, a task performed by the medical physicist. For more complex plans, such as those discussed here, several options may be prepared prior to selection and approval of the final plan by the clinician. In our examples, treatment plans using 3D-CRT and IMRT techniques were specified, prepared, evaluated and compared for a patient with a tumour located in the head and neck region and a patient with prostate cancer. An IMRT plan for a patient with arm cancer, untreatable with the 3D-CRT technique, is also discussed.

\section{Materials and methods}

\subsection{Clinical specification}

For the clinical case of head and neck cancer (right tonsil carcinoma) the organs at risk (OAR) are the spinal cord and larynx. In the 3D-CRT plan, the lower neck was to be treated with a static anterior field and the upper neck - with opposing lateral fields. Additional dose was to be delivered by opposing lateral electron fields to the posterior neck, to minimise the dose to the spinal cord. The IMRT treatment consisted of seven coplanar fields (Fig. 1). For treatment of subclinical disease, target volumes were to be treated by 60 Gy and by 54 Gy, in 30 daily fractions of 2 Gy.

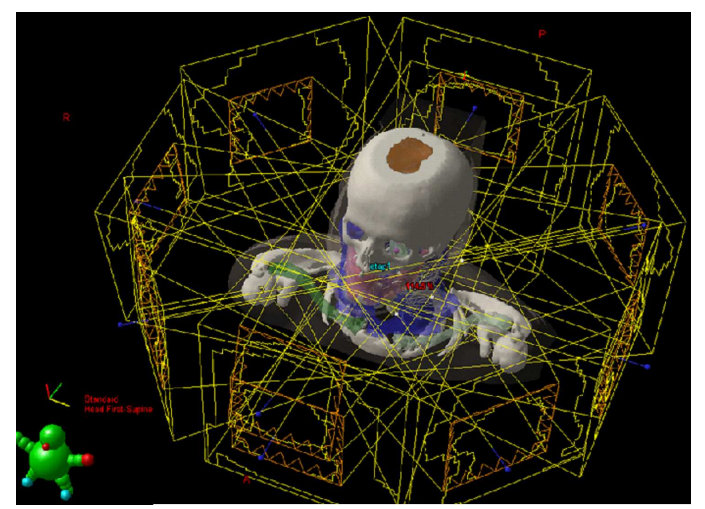

Fig. 1. Example of an IMRT head and neck treatment using seven coplanar fields.

Prostate cancer was treated in the second example. A total dose of $60 \mathrm{~Gy}$ was to be applied to the tumour volume in 20 daily fractions of 3 Gy each. Because of the tumour location, the main organs at risk were the rectum and the bladder. Four beams were used in the 3D-CRT plan (Fig. 2A) and five fields - in the IMRT technique (Fig. 2B).
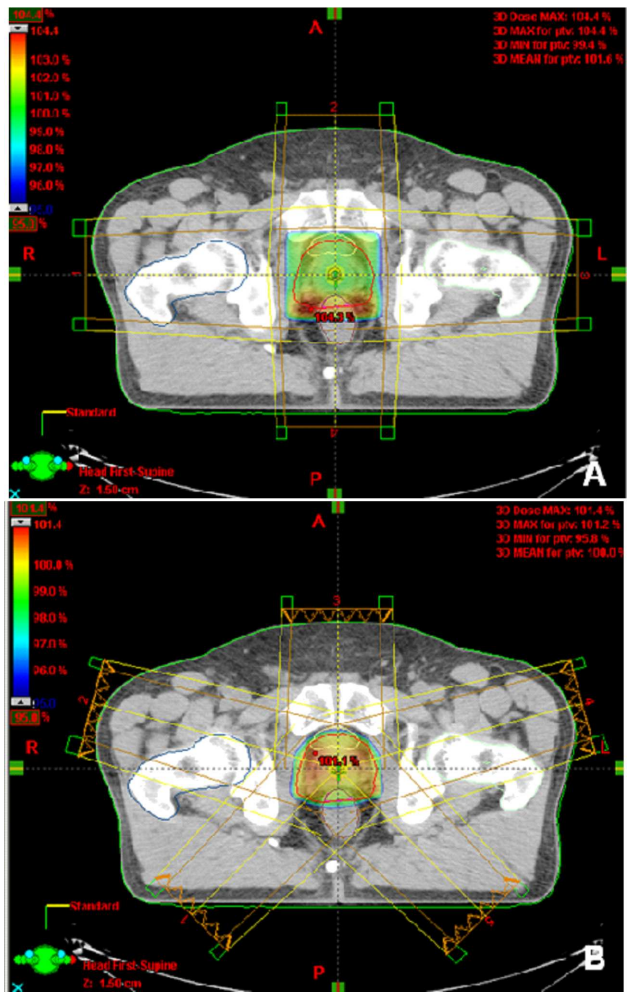

Fig. 2. Field configuration and transversal treatment plans for the prostate cancer patient treated with: (A) 3D-CRT and (B) IMRT techniques.

In the last example, of sarcoma of the left arm following gross resection, high dose gradients were required, to be accomplished using multiple coplanar IMRT fields with variable intensity. The prescribed dose to the tumour volume was $50 \mathrm{~Gy}$, delivered in 25 daily fractions of $2 \mathrm{~Gy}$ each.

\subsection{Treatment planning}

All patients were treated with $6 \mathrm{MV}$ or $18 \mathrm{MV}$ photon beams generated by a Varian $2300 \mathrm{C} / \mathrm{D}$ accelerator (Varian Medical Systems, Inc.) equipped with a Millennium MLC with 120 leaves (also from Varian). For treatment planning, CT scans with $2.5 \mathrm{~mm}$ step were performed. CT images were transferred to the Eclipse 7.3 planning system and tumour volumes and normal tissues including OAR were delineated by the physician-radiologist.

The gross tumour volume (GTV) consisted of the primary tumour and a number of involved lymph nodes. The clinical target volume (CTV) includes the GTV and sub-clinical nodal volumes. The planning target volume (PTV) consisted of the CTV with a $5 \mathrm{~mm}$ margin $[6,7]$. For 3D-CRT and IMRT planning, the PTV should receive at least $95 \%$ of the prescribed dose. Isodose distributions in three planes (axial, sagittal, and coronal) and 
dose-volume histograms (DVH) of PTV and OAR were used to evaluate the final treatment plans. The same target volumes, OAR and normal tissue of each patient were used to create the 3D-CRT plan and to generate the IMRT plan. In the 3D-CRT technique the number of treatment beams and appropriate beam geometries were selected and the dose distribution optimised by trial and error by the medical physicist. IMRT plans were generated using the ECLIPSE/HELIOS module which includes an inverse-planning algorithm [8]. Within this algorithm, the medical physicist specifies the required dose to the tumour volume and acceptable tolerance doses for the organs at risk, in the form of constraints. Then, the system arrives by itself at the best physical and technically possible treatment plan. In this work the best clinically acceptable 3D-CRT and IMRT plans were compared.

\subsection{Evaluation}

The isodose distribution was first compared visually on axial, sagittal and coronal slices with respect to the degree of conformity of the prescribed dose to PTV and delivery of the highest doses within OAR structures. Particular attention was paid to the $95 \%$, isodose (minimum therapeutic dose). The plan was also evaluated by comparing the cumulative DVH for planning target volumes and organs at risk.

The general shape and area under the DVH were instrumental in determining adequate coverage and homogeneity of dose in the target volume and within the OAR structures. The minimum and maximum dose to the PTV and the mean and maximum dose to OAR structures and normal tissues, were analysed. Target coverage was estimated by comparing the percentages of PTV volume covered by $95 \%$, or $100 \%$ of the prescribed dose (V95\% and V100\%, respectively).

\section{Results}

The 3D-CRT plan for the patient with prostate cancer is shown in Fig. 2A and the IMRT plan - in Fig. 2B. In the IMRT technique not only was the conformity of highdose volumes improved, but so were isodose distributions within normal tissues. The 3D-CRT and IMRT plans showed no significant differences in PTV regions, with the exception of maximum dose $\left(D_{\max }\right)$ for the PTV which was reduced from 59.6 to 57.4 Gy in the IMRT plan (Table I). In the IMRT technique, the mean dose $\left(D_{\text {mean }}\right)$ values for the rectum were reduced from 40.3 to $22.7 \mathrm{~Gy}$ (by $43 \%$ ) and for the bladder - from 45.4 to 26.2 Gy (by $42 \%$ ). In both IMRT and 3D-CRT plans comparable target coverage, with $100 \%$ of the PTV receiving $95 \%$ of the prescribed dose, was achieved.

The 3D-CRT and IMRT plans for the patient with tonsil cancer are shown in Fig. 3A and Fig. 3B, respectively. The IMRT technique gave better conformity of the prescribed isodose volume to PTV1 (60 Gy) and to PTV2 (54 Gy). Although the $D_{\max }$ of the PTV1 decreased slightly from 65.9 to $64.2 \mathrm{~Gy}$, target conformity

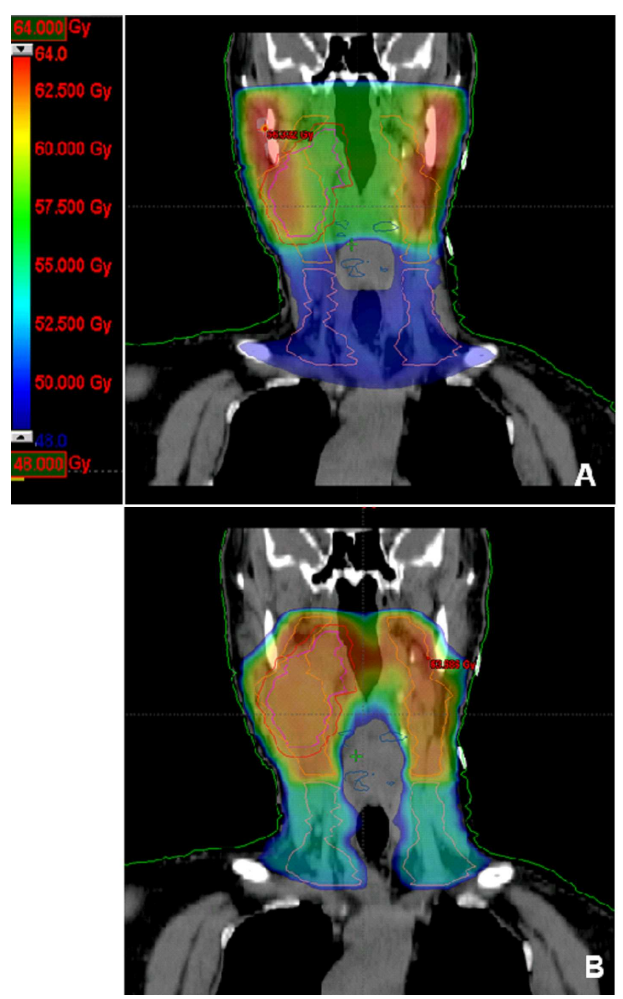

Fig. 3. Frontal isodose distributions for the patient with tonsil cancer treated with: (A) 3D-CRT and (B) IMRT techniques.

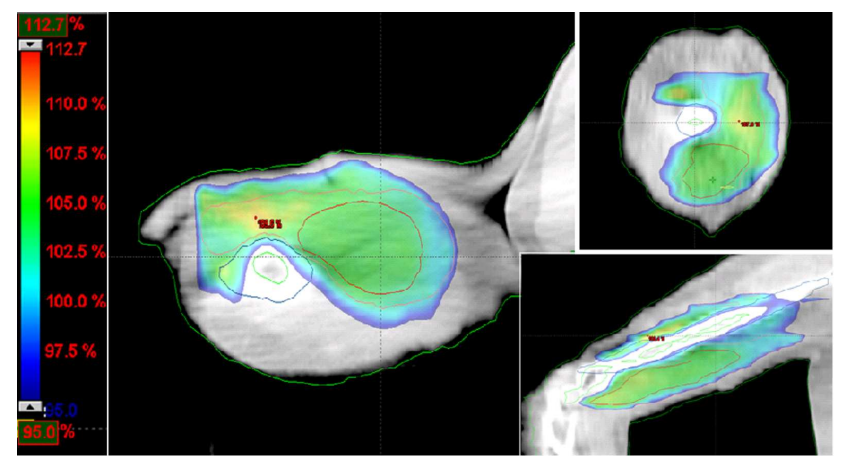

Fig. 4. Dose distributions in transversal, sagittal, and frontal projections for the patient with sarcoma of the left arm, treated with the IMRT technique.

improved due to $D_{\min }$ increasing from 39.6 to $57.6 \mathrm{~Gy}$ (Table II). Also the increased values of $D_{\min }$ and $D_{\max }$ for PTV2 gave very good coverage of the target. The $\mathrm{DVH}$ for the spinal cord and the larynx were improved in the IMRT plan, with $D_{\text {mean }}$ for spinal cord reduced from 28 to $13 \mathrm{~Gy}(53 \%)$ and $D_{\text {mean }}$ for the larynx from 45.3 to 37.9 Gy (16\%). The values of $\mathrm{V} 95 \%$ for PTV1 were $100 \%$ and $66.8 \%$ and for PTV $2-100 \%$ and $93.6 \%$ in the IMRT and 3D-CRT plans, respectively.

In the axial, sagittal, and coronal displays of the dose distribution for the patient with left arm sarcoma shown 
TABLE I

Target coverage and normal tissue sparing for the patient with prostate cancer.

\begin{tabular}{c|c|c}
\hline $\begin{array}{c}\text { Patient } \\
\text { (prostate) }\end{array}$ & $\begin{array}{c}\text { 3D-CRT } \\
{[\mathrm{Gy}]}\end{array}$ & $\begin{array}{c}\text { IMRT } \\
{[\mathrm{Gy}]}\end{array}$ \\
\hline PTV (50 Gy) & & \\
$D_{\min }$ & 59.6 & 57.4 \\
$D_{\max }$ & 62.7 & 61.7 \\
rectum & & \\
$D_{\text {max }}$ & 62.3 & 60 \\
$D_{\text {mean }}$ & 40.3 & 22.7 \\
bladder & & \\
$D_{\text {max }}$ & 61.6 & 60 \\
$D_{\text {mean }}$ & 45.4 & 26.2
\end{tabular}

TABLE II

Target coverage and normal tissue sparing for the patient with tonsil carcinoma.

\begin{tabular}{c|c|c}
\hline $\begin{array}{c}\text { Patient } \\
\text { (tonsil) }\end{array}$ & $\begin{array}{c}\text { 3D-CRT } \\
{[\mathrm{Gy}]}\end{array}$ & $\begin{array}{c}\text { IMRT } \\
{[\mathrm{Gy}]}\end{array}$ \\
\hline PTV (60 Gy) & & \\
$D_{\min }$ & 39.6 & 57.6 \\
$D_{\max }$ & 65.9 & 64.2 \\
PTV (54 Gy) & & \\
$D_{\min }$ & 45.5 & 50 \\
$D_{\max }$ & 51.6 & 59.6 \\
spinal cord & & \\
$D_{\max }$ & 44.4 & 38 \\
$D_{\text {mean }}$ & 28 & 13.7 \\
larynx & & \\
$D_{\max }$ & 63.5 & 62.9 \\
$D_{\text {mean }}$ & 45.3 & 37.9
\end{tabular}

in Fig. 4, the IMRT plan gave a conformal dose distribution within the target volume. While normal tissue tolerances were always exceeded in the 3D-CRT approach (results not shown), IMRT planning allowed the dose to be decreased to 23 Gy in bone tissue, and to 14.5 Gy in the bone marrow - the organ at risk in this case. It would not be possible to spare the bone marrow using the 3D-CRT approach.

\section{Discussion}

Modern external beam radiotherapy techniques were illustrated by a comparative evaluation of clinically acceptable 3D-CRT and IMRT plans prepared in our Centre of Oncology for treating patients with tumours in different localisations.

In general, our results are comparable with those published in literature [9].
Radiotherapy of the prostate can either be accomplished with IMRT or using a few (usually four) static fields. However, some authors have suggested use of IMRT technique to reduce the mean dose to the rectum and bladder [10, 11]. For head and neck cancer, the IMRT technique may further decrease the dose to selected organs at risk outside the target volume and thereby enable dose escalation $[12,13]$.

In many cases intensity modulated radiotherapy for soft tissue sarcoma (arm cancer) is strongly recommended. This technique can reduce the dose to bone marrow without compromising target coverage by achieving concave dose distributions around the interface of the PTV and bone $[14,15]$.

Comparison of dose distributions showed that with the IMRT technique target dose homogeneity was improved and that the dose to normal tissues and OAR structures was reduced. Significant reductions in the mean and maximum doses to the spinal cord (head and neck) and rectum (prostate) were also achieved. With respect to the PTV, IMRT planning increased the minimum while reducing the maximum dose, resulting in improved conformity of the high-dose volume to the tumour volume. As for geometrical issues, application of the IMRT technique clearly resulted in improvement of the dose distribution in all patients, especially where concave distributions were required.

IMRT enables dose escalation because of the unique ability of IMRT to create dose distributions that are physically superior to plans developed by standard 3-dimensional conformal techniques. Multiple targets and boost fields can be integrated into a single treatment plan, thus reducing the number of fractions required. While this may suggest lower cost of treatment, the preparation of IMRT plans is much more laborious and demands on its delivery to the patient are much more stringent than those of 3D-CRT. In our examples, with carefully selected beam parameters, IMRT improved target conformity, also allowing better sparing of critical structure and reduction of integral dose to be achieved. It should be stressed however that the type of radiotherapy equipment and the definition of the target volume may have considerable impact on the dose distributions resulting from the inverse planning process, and on the final effect of the treatment. Conclusive estimates of the risk of potential complications from a small volume receiving a high dose against that of a large volume receiving low dose, are still unknown. Therefore the accuracy and repeatability of patient positioning and immobilisation may crucially affect the outcome of IMRT treatment, it should therefore be closely observed and controlled through stringent Quality Assurance procedures.

\section{Acknowledgments}

A.Ś. gratefully acknowledges Dr. Jan Lesiak and Prof. Michael Waligórski for advice and help in presenting this work. 


\section{References}

[1] S. Webb, The Physics of Three-Dimensional Radiation Therapy: Conformal Radiotherapy, Radiosurgery and Treatment Planning, Institute of Physics Publishing, Bristol 1993.

[2] S. Webb, The British J. Radiol. 76, 678 (2003).

[3] W. Schlegel, A. Mahr, 3D Conformal Radiation Therapy - Multimedia Introduction to Methods and Techniques, Multimedia CD-ROM Springer, New York 2001.

[4] New Technologies in Radiation Oncology, Eds. W. Schlegel, T. Bortfeld, A.-L. Grosu, Springer, New York 2006

[5] K.S. Chao, S. Apisarnthanarax, G. Ozyigit, Practical Essentials of Intensity Modulated Radiation Therapy, 2nd ed., Lippincott, Williams \& Wilkins, Philadelphia 2005.

[6] ICRU, Prescribing, Recording and Reporting Photon Beam Therapy, Report 50, International Commission on Radiation Units and Measurements, Washington, DC 1993.

[7] ICRU, Prescribing, Recording and Reporting Photon Beam Therapy (Supplement to ICRU Report 50), Report 6, International Commission on Radiation Units and Measurements, Washington, DC 1999.
[8] S. Bin, Y. Shiao, The Oncologist 4, 422 (1999).

[9] Image Guided IMRT, Eds. T. Bortfeld, R. Schmidt-Urlich, W. de Neve, D.E. Wazer, Springer, Berlin 2006.

[10] M. Guckenberger, F. Pohl, K. Baier, J. Meyer, D. Vondermark, M. Flentje, Radiother. Oncol. $\mathbf{7 9}$, 59 (2006).

[11] C. Fiorino, C. Gianolini, A.E. Nahum, Phys. Med. Biol. 48, 2603 (2003)

[12] B. Longobardi, E. De Martin, C. Fiorino, I. Dell'oca, S. Broggi, G. Cattaneo, R. Calandrino, Radiother. Oncol. 77, 148 (2005)

[13] W. Bar, M. Shwarz, M. Alber, B.J. Mijnheer, C. Rash, C. Schneider, F. Nusslin, E.M. Damen, $R a-$ diother. Oncol. 69, 251 (2003).

[14] K.M. Alektiar, L. Hong, M.F. Brennan, C. Della-Biancia, S. Singer, Int. J. Radiation Oncology Biol. Phys. 68, 458 (2007).

[15] L. Hong, K.M. Alektiar, M. Hunt, E. Venkatraman, S. Leibel, Int. J. Radiation Oncology Biol. Phys. 59, 752 (2004). 